\title{
A NOTE ON GREEN'S THEOREM
}

B. D. CRAVEN

(received 31 October 1963)

Green's theorem, for line integrals in the plane, is well known, but proofs of it are often complicated. Verblunsky [1] and Potts [2] have given elegant proofs, which depend on a lemma on the decomposition of the interior of a closed rectifiable Jordan curve into a finite collection of subregions of arbitrarily small diameter. The following proof, for the case of Riemann integration, avoids this requirement by making a construction closely analogous to Goursat's proof of Cauchy's theorem. The integrability of $Q_{x}-P_{v}$ is assumed, where $P(x, y)$ and $Q(x, y)$ are the functions involved, but not the integrability of the individual partial derivatives $Q_{x}$ and $P_{y}$; this latter assumption being made by other authors. However, $P$ and $Q$ are assumed differentiable, at points interior to the curve.

Theorem. Let $C$ be a closed rectifiable Jordan curve, enclosing a plane region $R$. Let the functions $P(x, y)$ and $Q(x, y)$ be differentiable at all points of $R$, and continuous on $W=C+R$. Let $Q_{x}-P_{y}$ be Riemann-integrable on $R$. Then

$$
\int_{C}(P d x+Q d y)=\iint_{R}\left(Q_{x}-P_{y}\right) d x d y .
$$

Proof. Let $C$ have finite positive length $L$. Then there is a square $A$ of area $L^{2}$, with sides parallel to the axes, which contains $W$.

Choose any positive $\varepsilon$. In what follows, a neighbourhood of a point shall denote a square neighbourhood, with the point as its centre, and with sides parallel to the axes. Then, from the hypotheses, every point $\left(x_{0}, y_{0}\right)$ of $R$ has a neighbourhood $N\left(x_{0}, y_{0}\right)$ such that, for every point $(x, y)$ which lies in both $N$ and $W$,

$$
\begin{gathered}
\left|P(x, y)-P\left(x_{0}, y_{0}\right)\right|<\frac{1}{2} \varepsilon / L \\
\left|Q(x, y)-Q\left(x_{0}, y_{0}\right)\right|<\frac{1}{2} \varepsilon / L \\
P(x, y)=P^{*}+P_{x}^{*}\left(x-x_{0}\right)+P_{y}^{*}\left(y-y_{0}\right)+\xi \\
Q(x, y)=Q^{*}+Q_{x}^{*}\left(x-x_{0}\right)+Q_{y}^{*}\left(y-y_{0}\right)+\eta
\end{gathered}
$$

where $P^{*}, P_{x}^{*}, P_{y}^{*}, Q^{*}, Q_{x}^{*}, Q_{v}^{*}$ denote the values of $P, P_{x}, P_{y}, Q, Q_{x}$, $Q_{y}$ at $\left(x_{0}, y_{0}\right)$, and 


$$
\begin{aligned}
& |\xi|<\varepsilon r / L^{2} \\
& |\eta|<\varepsilon r / L^{2}
\end{aligned}
$$

where

$$
r^{2}=\left(x-x_{0}\right)^{2}+\left(y-y_{0}\right)^{2} .
$$

Divide $A$ into four squares of side $\frac{1}{2} L$, by lines parallel to the axes. Repeat this procedure for each of the four squares, and so on indefinitely. Denote by $F$ the family of closed squares, with sides tending to zero, so obtained. Denote by $F(\delta)$ the subset of $F$ consisting of squares each of side $\delta$, for $\delta=\frac{1}{2} L, \frac{1}{4} L, \cdots$. In this notation, Lemma 2 of Potts [2] states that the number of squares of $F(\delta)$ necessary to cover $C$ is less than $4(L / \delta)+4$. This follows, since an arc of $C$ of length less than $\delta$ can have points in common with at most four such squares.

There exists, for some $\delta$, a finite collection $F_{1}$ of squares $A_{i}$ of $F$, disjoint except for common boundaries, such that every point of $W$ lies in some $A_{i}$, and such that if $A_{i}$ lies wholly interior to $R$, and $\left(x_{0}, y_{0}\right)$ is its centre point, then every point $(x, y)$ of $A_{i}$ satisfies (2) to (7), whereas if $A_{i}$ contains points of $C$, then $A_{i}$ belongs to $F(\delta)$, and (2) and (3) hold for any two points $\left(x_{0}, y_{0}\right)$ and $(x, y)$ which lie in both $A_{i}$ and $W$. For if not, some region of $W$ requires an infinite collection of squares. Then successive subdivision of this region produces a nested sequence of squares, to each of which the same statement applies. Since $W$ is compact, the nested sequence defines a limit point in $W$, at which $P$ or $Q$ is discontinuous or not differentiable, contrary to hypothesis.

Let $F^{\prime}$ denote any finite collection of squares of $F$, obtained by further subdividing the squares of $F_{1}$. The relation between $F^{\prime}$ and $F_{1}$ will be written $F^{\prime}<F_{1}$. Then if $F_{1}$ has the property stated in the previous paragraph, the same statement applies to any $F^{\prime}<F_{1}$.

Denote by $R^{*}$ the union of those squares of $F_{1}$ which contain points of $W$. Let

$$
\begin{aligned}
\phi(x, y) & =Q_{x}(x, y)-P_{y}(x, y) \text { for }(x, y) \text { in } W \\
& =0 \text { elsewhere. }
\end{aligned}
$$

Then

$$
\iint_{R}\left(Q_{x}-P_{y}\right) d x d y=\iint_{R^{*}} \phi d x d y
$$

Since by hypothesis, this Riemann integral exists, there exists, for some $\delta<\varepsilon /(L+1)$, a finite collection $F_{2}$ of squares of $F(\delta)$, with $F_{2}<F_{1}$, such that

$$
\left|\iint_{R^{*}} \phi d x d y-\Sigma\left(Q_{x}^{*}-P_{y}^{*}\right)\right| A_{i}||<\varepsilon
$$


where $Q_{x}^{*}$ and $P_{y}^{*}$ refer to the centre point of $A_{i}$, and $\left|A_{i}\right|$ is the area of $A_{i}$. The summation includes all squares $A_{i}^{\prime}$ of $F_{2}$ which lie wholly within $R$, and some (possibly all or none) of those squares $A_{i}^{\prime \prime}$ which include points of $C$. Let $B$ denote an upper bound to $\phi$, implied by its Riemann-integrability. Then

$$
\left|\Sigma\left(Q_{x}^{*}-P_{y}^{*}\right)\right| A_{i}^{\prime \prime}||<B \sum\left|A_{i}^{\prime \prime}\right|
$$

By Pott's Lemma,

$$
\sum\left|A_{i}^{\prime \prime}\right| \leqq \delta^{2}[4(L / \delta)+4]<4 \varepsilon
$$

supposing $\varepsilon<1$. Therefore

$$
\left|\iint_{R}\left(Q_{x}-P_{y}\right) d x d y-\sum\left(Q_{x}^{*}-P_{y}^{*}\right)\right| A_{i}^{\prime \prime}||<(1+4 B) \varepsilon .
$$

Denote by $\rho_{i}^{\prime}$ the boundary of $A_{i}^{\prime}$, and by $\rho_{i}^{\prime \prime}$ the boundary of that part of $A_{i}^{\prime \prime}$ which lies in $W$. Then if $\left|\rho_{i}^{\prime \prime}\right|$ denotes the length of $\rho_{i}^{\prime \prime}$,

$$
\sum\left|\rho_{i}^{\prime \prime}\right|<L+(4 \delta)[4(L / \delta)+4]<17(L+1)
$$

again applying Potts' Lemma. Now

$$
\int_{C}(P d x+Q d y)=\Sigma \int_{\rho_{i}^{\prime}}(P d x+Q d y)+\sum \int_{\rho_{i}^{\prime \prime}}(P d x+Q d y)
$$

with all paths traversed in the positive direction. From (4) and (5),

$$
\int_{\rho_{i}^{\prime}}(P d x+Q d y)=\left(Q_{x}^{*}-P_{v}^{*}\right)\left|A_{i}\right|+\int_{\rho_{i}^{\prime}}(\xi d x+\eta d y),
$$

since for a square,

$$
-\int_{\rho_{i}^{\prime}} y d x=\left|A_{i}^{\prime}\right| \text { and } \int_{\rho_{i}} x d y=\left|A_{i}^{\prime}\right|
$$

By (6) and (7),

$$
\Sigma\left|\int_{\rho_{i}^{\prime}}(\xi d x+\eta d y)\right| \leqq \Sigma\left(\varepsilon \mid L^{2}\right) \cdot 4 \sqrt{ } 2\left|A_{i}^{\prime}\right|<6 \varepsilon
$$

And from (2) and (3), and (12),

$$
\Sigma\left|\int_{\rho_{i}^{\prime \prime}}(P d x+Q d y)\right|<(\varepsilon / L) \cdot 17(L+1)=B^{\prime} \varepsilon, \text { say. }
$$

Combining (11), (13), (14), (15), and (16),

$$
\left|\iint_{R}\left(Q_{x}-P_{y}\right) d x d y-\int_{C}(P d x+Q d y)\right|<\left(7+4 B+B^{\prime}\right) \varepsilon .
$$

Since $\varepsilon$ is arbitrary, (1) is proved. 


\section{Acknowledgement}

Thanks are due to Professor E. R. Love, whose suggestion it was that Goursat's method might be applicable to Green's Theorem, and to the referees for several suggestions.

\section{References}

[1] Verblunsky, J., On Green's formula, J. Lond. Math. Soc., 24 (1949), 146-148.

[2] Potts, D. H., A note on Green's Theorem, J. Lond. Math. Soc., 26 (1951), 302-304.

Department of Mathematics,

University of Melbourne. 